\title{
Effectiveness of extracorporeal shockwave therapy for frozen shoulder in perimenopausal diabetic women
}

\author{
Radwa Mohamed Yehia ${ }^{1}$, Mohamed M. ElMeligie ${ }^{2}$ \\ 1 Department of Physical Therapy for Obstetrics and Gynecology, Faculty of Physical Therapy, October 6 University, \\ Egypt; ${ }^{2}$ Department of Basic Sciences, Faculty of Physical Therapy, October 6 University, Egypt
}

\begin{abstract}
Study aim: To evaluate the effectiveness of extracorporeal shockwave therapy (ESWT) for frozen shoulder in perimenopausal diabetic women.

Materials and methods: A single blind randomized controlled trial was conducted on sixty-two perimenopausal women diagnosed with diabetic frozen shoulder. They were divided randomly into two groups. Group (A) received physiotherapy program in the form of maitland joint mobilization for shoulder joint, self-stretching exercises, range of motion exercises (ROM) and strengthening exercises. Sessions were held 3 times per week, for 4 weeks. Group (B) study group: received the same physiotherapy program in addition to ESWT, once per week, for 4 weeks. Visual analogue scale was used to measure pain intensity, shoulder pain and disability index (SPADI) was used to evaluate disability level, while an electrogoniometer was used to measure shoulder flexion, abduction and external rotation range of motion. All measurements were recorded pretreatment and posttreatment program.

Results: For intragroup comparison, significant improvement in all treatment outcomes was noted in both groups $(\mathrm{P}<0.05)$. However, no significant differences were found between both groups regarding all outcome measures in case of intergroup comparison in the study $(\mathrm{P}>0.05)$.

Conclusion: The use of ESWT have a positive effect in reducing pain and improving shoulder range of motion in perimenopausal women suffering from frozen.
\end{abstract}

Key words: Extracorporeal shock wave therapy - Frozen shoulder - Diabetes - Perimenopausal women

\section{Introduction}

Adhesive capsulitis (AC) is common spread within women of forty to seventy years. Frozen shoulder (FS) is another name of $\mathrm{AC}$, it is an idiopathic problem affecting the musculoskeletal system causing pain and limitation in shoulder movement [10, 32]. It disrupts patient's daily life due to degenerative changes that develops in the joint capsule with associated adhesions, rotator cuff, biceps brachii and the surrounding tissues [33]. This will eventually lead to a decline in external rotation and loss of flexibility [14]. Adhesive capsulitis (AC) usually develops by 3 phases: Freezing (painful) phase: it is length two to nine months and pain increased at night. Frozen (adhesive) phase: the pain will begin to subside followed by gradual limitation in shoulder movement in all directions for four to twelve months and Thawing phase: in this phase there is progressive go back to range of motion (ROM) that takes about 5-26 months to complete [8].

Diabetes Mellitus (DM) increasing hardship of FS patients because it affects their musculoskeletal system which aggravates pain and ROM limitations in both shoulders [37]. There is a variety of definitions regarding the term perimenopause found in the previous literature, this paper will use the definition suggested by several established organizations such as the Society of Obstetricians and the World Health Organization (WHO), which state that perimenopause starts from two to eight years before menopause and the first year after the last menstrual period. According to this definition, perimenopause usually develops around the fifth decade due to the endocrinological and clinical disturbances that accompanies the actual menopause [5]. 
Dedicação et al., reported that $70 \%$ of perimenopausal women suffer from symptoms associated with estrogen deficiency such as musculoskeletal pain [13]. Around time of menopause (perimenopause), estrogen deficiency takes place causing slow-progressing joint inflammation (osteoarthritis) which leading to cartilage degeneration [20]. A study performed by Yokota et al., explored the relation between symptoms severity and body mass index in Japanese middle-aged women, they concluded that shoulder stiffness is a characteristic symptom in Japanese perimenopausal women [41].

A possible explanation for musculoskeletal pain in the perimenopausal period can be related to hormone alterations, especially hypoestrogenism, that can cause the wearing of the cartilage, like the bone mass loss that occurs in perimenopausal women. On the other hand, the sexual hormones are part of the pain modulation process, which makes perimenopausal women more sensitive to pain. [13].

There are many localized physiological effects of shockwave in diabetic FS through enhancing the local circulation, facilitate neovascular changes, production of enzymes and enhance local flexibility of collagen fibers because of decreased secretion of inflammatory cytokines $[1,21]$. Pain arising from musculoskeletal disorder such as myofascial pain, rotator cuff tendonitis and sacroiliac joint pain can be controlled by ESWT due to is powerful analgesic effects [7].

The purpose of this study is to evaluate the difference in effectiveness between ESWT and standard physiotherapy for frozen shoulder in perimenopausal women. It was hypothesized that there would be a significant difference between ESWT with maitland joint mobilization for shoulder joint, self-stretching exercises, range of motion exercises (ROM) and strengthening exercises would minimize pain and improve ROM and function in perimenopausal diabetic women with frozen shoulder.

\section{Materials and methods}

This study was conducted at the outpatient clinic of October 6 University hospital between November 2020 and January 2021. Sixty-two women diagnosed with diabetic frozen shoulder based on MRI findings and clinical examination were recruited for this study. COVID safety measures were taken during the study as described by World Health Organization [40]. The perimenopausal status of participants was identified as described previously $[8,11,26]$. The inclusion criteria were: (1) Women's age between 40 to 60 years old; (2) suffering from AC for 2-9 months (stage 1: the painful phase) confirmed by an orthopedic specialist; (3) passive/ active restricted ROM less than or equal to 90 degrees at external rotation, abduction, and flexion; (4) diagnosed as type $2 \mathrm{DM}$ for at least 5 years (FBS $\geq 126 \mathrm{mg} / \mathrm{dl}$ ); (5) no treatment other than analgesics. Patients were excluded from the study were cases of traumatic frozen shoulder, rheumatoid arthritis, recurrent subluxation of shoulder, cervical radiculopathy, history of shoulder surgery, history of tumors, and vascular diseases. The study followed the Declaration of Helsinki guidelines and received the ethical permission from the local institutional review board at the Faculty of Physical Therapy, Cairo University under registration number P.T.REC/012/002983 before the study commencement. In addition, all subjects signed a written informed consent regarding use of anonymized data for research and publication purposes.

\section{Sample size determination}

A preliminary power analysis ( power $=0.8 ; \alpha=0.05$ ) determined an appropriate sample size of at least 58 cases based on a previous similar study [35].

\section{Study design}

This study was designed as a prospective, randomized, controlled trial. Total number of participants were 70 perimenopausal women, 3 participants did not meet the inclusion criteria, five perimenopausal women refused to participate in this study and 62 perimenopausal women were included in the study (Figure 1). An orthopedic surgeon assessed the participants for eligibility to the study, and he was blinded to the study. All the participants were assessed by the same physician prior to beginning the program and after the 4 weeks finished. The patients were not informed of the treatment assignments. The second author who was not involved in data collection process randomized all patients who met the inclusion criteria using sequentially numbered opaque sealed envelopes that contained treatment assignments drawn from a random number software generator. An independent researcher was recruited for for opening the opaque envelopes. A researcher different from $1^{\text {st }}$ and $2^{\text {nd }}$ authors belonging to our research group was responsible for data collection at baseline and after the end of the treatment program, and he was blinded to group allocation.

Patients who fulfilled the inclusion criteria were randomly assigned to one of 2 groups using a secure system of opaque closed envelopes that were arranged from A to B. Patients were assigned to one of the 2 groups: The control group (Group A) included 31 diabetic female (FBS $\geq$ $126 \mathrm{mg} / \mathrm{dl}$ ) with shoulder AC who received physiotherapy for 4 weeks while the study group (Group B) included 31 diabetic female (FBS $\geq 126 \mathrm{mg} / \mathrm{dl}$ ) with shoulder AC whom get a similar physiotherapy program, together with ESWT for 4 weeks. 


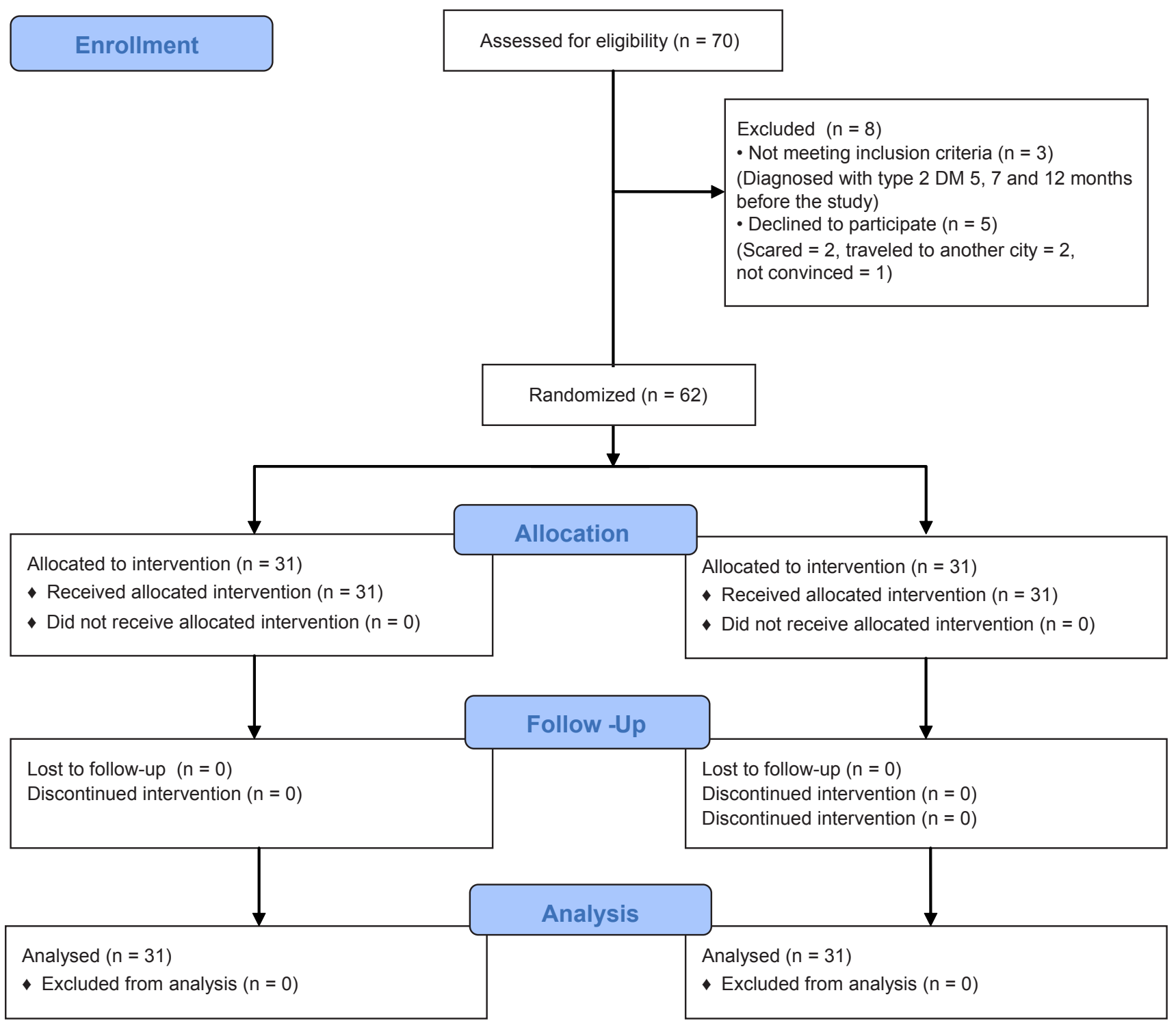

Figure 1. Flowchart showing the experimental design of the study

\section{Intervention}

\section{Group (A):}

Participants in this group received physiotherapy program in the form of: (a) Maitland passive joint mobilization for shoulder joint; (b) self-stretching exercises; (c) ROM exercises in pain-free range for 5-10 minutes; (d) strengthening exercises [36]. Two certified instructors supervised the exercises as a daily home routine with video calls. The instructors were blinded to the study.

\section{A. Maitland's Mobilization procedure:}

When participants in supine lying position, distraction of the humeral head was applied at $1^{\text {st }}$, before mobilization. To mobilize the anterior capsule's compartment, shoulder joint in $90^{\circ}$ flexion, then press the posterior shoulder compartment, the applied force was toward the anterior direction. To mobilize the anterior capsule's compartment, shoulder $90^{\circ}$ abduction, then press the anterior part of the shoulder, the applied force was toward the posterior direction. Longitudinal caudal mobilization was indicating diabetic woman to abduct her shoulder joint to $90^{\circ}$. The accessory mobilization was performed at a rate of two to three passive oscillatory movements per second for approximately 30 seconds, 5 sets were applied [25, 31].

B. Stretching exercises:

Self-stretching Exercises were started in low intensity for all shoulder joint directions aiming to increase ROM of glenohumeral joint. Participants were asked to maintain each stretch for 30 seconds followed by 10 seconds rest period in between and were repeated for 5 times. Participants were practiced stretching exercises as a daily home routine [4, 27]. For shoulder external rotation and flexion stretching, woman was seated on a stool at the side of a treatment table with her forearm rested on the treatment table and her elbow flexed $90^{\circ}$, lean 
forward until her head and shoulder reach to the treatment table level.

C. Range of Motion Exercises Program:

- Finger ladder Exercises: a hanging ladder over a wall was used in which a woman stood facing it. The affected hand rested on the ladder at starting step at a low level and moved slowly toward the above steps as much as she can, then slowly moved down toward the below steps until reach the starting step.

- Circumduction Exercises: women were assumed a prone lying toward the edge of the bed, while the affected shoulder hanged out of the bed then slowly rotate it in circular motion in form of sets for 5 to 10 times/day.

- Pendulum Exercises: from sitting on stool women were leaned toward the treatment table with non-affected side supported on it, while the affected side shacked several times to reach complete relaxation then move it forward and backward several times until feel the full weight of the arm with slight stretching with pain free in form of sets for 5 to 10 times/day.

- Pulley Exercises:

$1^{\text {st }}$ shoulder pulley exercise (Up and Down - Facing the shoulder rope pully): woman's chair faced the shoulder rope pully, the non-affected arm pulls the injured arm, gently stretching it forwards and up, holden in the final position for 10 seconds and then lower the arm.

$2^{\text {nd }}$ shoulder pulley exercise (Shoulder Abduction): the chair was turned sideways to the shoulder rope pully so the injured arm is closest to the door. The pulley handles were holden and pulled the injured arm up to the side as high as possible alternatively with the non-affected side. Hold for 10 seconds. Relax and repeat 5 to 10 times.

$3^{\text {rd }}$ shoulder pulley exercise (Up and Down Back to the shoulder rope pully): the chair was turned to facing the rope pully. The pulley handles were holden and pulled the injured arm up to the side as high as possible alternatively with the non-affected side. Hold for 10 seconds. Relax and repeat 5 to 10 times.

$4^{\text {th }}$ shoulder pulley exercise (Internal Rotation): the woman was seated on a chair against the shoulder rope pully. Woman was grasped the pulley handles with both hands. Pulled down on the pulley with non-affected arm. This moved up the affected arm over woman's head as high as she can. Hold for 10 seconds. Relax and repeat 5 to 10 times [36].

D. Strengthening exercises:

Strengthening exercises were started with weights, therabands and springs .The exercise protocol and manual therapy were given to improve coordination, muscle strength and mobility of rotator cuff muscles to unload the subacromial space during active movements.

Group (B):

All patients in both groups received the same physiotherapy program in addition to ESWT, once per week, for
4 weeks. The treatment position was sitting with the full support of the back; the affected shoulder was exposed, and a coupling gel was applied on it to limit the loss of energy at the interface between skin and applicator head. Then, the ESWT was applied close to the insertion of rotator cuff muscle on the most trigger point (subscapularis muscle) in accordance with the previous findings [18]. The ESWT applied 2000 impulses in one session. The density of energy was $0.22 \mathrm{~mJ} / \mathrm{mm}^{2}$, the pulse rate was $10 / \mathrm{sec}$ and the frequency was $1-15 \mathrm{~Hz}$ [3].

\section{Outcome measures}

1. Visual analogue scale (VAS) was used to assess the severity of pain before and after treatment for both groups. It consists of a $10 \mathrm{~cm}$ line with varying degrees of pain intensity from 0 (no pain) to 10 (killing pain). Patients were instructed to place a mark along the line to indicate their pain severity [15].

2. Shoulder pain and disability index (SPADI) was used for evaluation of pain and functional disability of the shoulder joint pre/post-treatment for both groups. It is a self-revealed measure created to assess shoulder pathological conditions. It exhibits good test-retest reliability \& construct and criterion related validity. The index consists of 13 items that represent two subscales: pain level (which compromising 5 items and functional disability compromising 8 items. Each item in the pain subscale offered 11 answers, ranging from zero (representing no pain state) to 10 (representing the worst imaginable pain). Every item in the disability subscale was presented with 11 answers that ranges from zero (no difficulty) to 10 (difficulty requiring assistance). The overall score was calculated by averaging the scores of pain and disability subscales [38].

3. Electrogoniometer [Model 01129 Guymon goniometer] was used to measure range of moton for all patients in both groups pre- and post-treatment. The assessment was done for shoulder flexion, abduction and external rotation for every patient. Three continuous measures were recorded in each direction and the average of them was taken [12].

\section{Statistical analysis}

Statistical analysis was done by a statistician who was blinded to the treatment and group's allocation. Normal distribution of data was checked using the Shapiro-Wilk test for all variables. The mean and standard deviation (SD) were used for expression of results for data analysis. Unpaired t-test was used to compare various variables between groups. Paired t-test was performed within same group for various variables as compare pre- and postassessment in normal data. Statistical Package for Social Sciences (SPSS) computer program (version 23 windows) 
was used to analyze the data. The significance of effects was pre-determined at $\leq 0.05$.

\section{Results}

Sixty two patients were recruited for the study. No adverse events were recorded at any time during the study period, and all patients completed the assigned treatment and were analyzed at the end of the study. The demographic and clinical baseline characteristics of the three groups were well balanced (Table 1).

The results of VAS, SPADI, shoulder flexion, abduction, and external rotation ROM for the two groups at each measurement stage are shown in table 2. For intragroup comparison, both the control group and study group showed significant differences between pre- and posttreatment scores for all outcome's measures.

Table 1. Descriptive statistics and independent $t$ tests for the participants in both groups

\begin{tabular}{|c|c|c|c|c|c|}
\hline Parameters & Group A & Group B & t value & $\mathrm{p}$ value & Sig. \\
\hline Age [years] & $52.7 \pm 7.8$ & $52.4 \pm 7.1$ & -0.328 & 0.745 & NS \\
\hline Weight $[\mathrm{kg}]$ & $75.68 \pm 6.83$ & $78.26 \pm 7.54$ & 1.4124 & 0.163 & NS \\
\hline Height $[\mathrm{cm}]$ & $172.87 \pm 4.51$ & $173.65 \pm 3.89$ & 0.7243 & 0.4717 & NS \\
\hline Body mass index (BMI) $\left[\mathrm{kg} / \mathrm{m}^{2}\right]$ & $24.568 \pm 2.542$ & $25.729 \pm 2.662$ & 1.7568 & 0.0841 & NS \\
\hline Systolic blood pressure $[\mathrm{mmHg}]$ & $108.5 \pm 10.7$ & $106.9 \pm 11.1$ & 0.5778 & 0.5656 & $\mathrm{NS}$ \\
\hline Diastolic blood pressure $[\mathrm{mmHg}]$ & $73.7 \pm 6.58$ & $76.2 \pm 4.4$ & 1.7585 & 0.0838 & NS \\
\hline Time since first diagnosis of frozen shoulder (FS) [years] & $8.2 \pm 2.4$ & $7.4 \pm 1.59$ & 1.5472 & 0.1271 & NS \\
\hline Duration of illness (FS) [months] & $6.3 \pm 2.58$ & $6.1 \pm 2.32$ & 0.3209 & 0.7494 & NS \\
\hline
\end{tabular}

Table 2. The VAS, SPADI and shoulder ROM for both groups

\begin{tabular}{|c|c|c|c|c|}
\hline Parameter & & Control group & Study group & P value* \\
\hline \multirow{4}{*}{ VAS } & Pretreatment & $7.9 \pm 1.2$ & $7.6 \pm 1.34$ & $0.22 \mathrm{~ns}$ \\
\hline & Posttreatment & $2.8 \pm 0.92$ & $1.9 \pm 0.73$ & $0.08 \mathrm{~ns}$ \\
\hline & $\%$ of improvement & $64.55 \%$ & $75.0 \%$ & \\
\hline & $\mathrm{P}$ value ** & $0.001 \mathrm{~S}$ & $0.001 \mathrm{~S}$ & \\
\hline \multirow{4}{*}{ SPADI } & Pretreatment & $7.4 \pm 1.2$ & $6.92 \pm 1.3$ & $0.36 \mathrm{~ns}$ \\
\hline & Posttreatment & $1.9 \pm 0.85$ & $1.5 \pm 0.68$ & $0.1 \mathrm{~ns}$ \\
\hline & $\%$ of improvement & $74.3 \%$ & $78.3 \%$ & \\
\hline & $\mathrm{P}$ value $* *$ & $0.001 \mathrm{~S}$ & $0.001 \mathrm{~S}$ & \\
\hline \multirow{4}{*}{ SHOULDER FLEXION } & Pretreatment & $94.35 \pm 6.38$ & $96.79 \pm 6.87$ & $0.47 \mathrm{~ns}$ \\
\hline & Posttreatment & $138.52 \pm 12.3$ & $145.2 \pm 11.15$ & $0.16 \mathrm{~ns}$ \\
\hline & $\%$ of improvement & $46.86 \%$ & $50.01 \%$ & \\
\hline & $\mathrm{P}$ value ** & $0.001 \mathrm{~S}$ & $0.001 \mathrm{~S}$ & \\
\hline \multirow{4}{*}{ SHOULDER ABDUCTION } & Pretreatment & $103.53 \pm 7.98$ & $102.1 \pm 7.63$ & $0.1 \mathrm{~ns}$ \\
\hline & Posttreatment & $154.66 \pm 20.32$ & $159.5 \pm 10.97$ & $0.54 \mathrm{~ns}$ \\
\hline & $\%$ of improvement & $49.32 \%$ & $56.3 \%$ & \\
\hline & $\mathrm{P}$ value ** & $0.001 \mathrm{~S}$ & $0.001 \mathrm{~S}$ & \\
\hline \multirow{4}{*}{$\begin{array}{l}\text { SHOULDER EXTERNAL } \\
\text { ROTATION }\end{array}$} & Pretreatment & $23.33 \pm 4.57$ & $24.6 \pm 4.26$ & $0.28 \mathrm{~ns}$ \\
\hline & Posttreatment & $38.2 \pm 6.21$ & $42.33 \pm 2.44$ & $0.35 \mathrm{~ns}$ \\
\hline & $\%$ of improvement & $63.3 \%$ & $72.07 \%$ & \\
\hline & $\mathrm{P}$ value $* *$ & $0.001 \mathrm{~S}$ & $0.001 \mathrm{~S}$ & \\
\hline
\end{tabular}

* Inter-group comparison; ** intra-group comparison of the results pre- and post-treatment. $\mathrm{ns}-\mathrm{P}>0.05=$ non-significant, $\mathrm{SP}<0.05=$ significant, $\mathrm{P}=$ probability. 
For Intergroup comparisons, at baseline, no differences were detected between the two groups in pain $(p=0.22)$, SPADI $(p=0.36)$, shoulder flexion ROM $(p=0.47)$, shoulder abduction ROM ( $p=0.54)$ or shoulder external rotation ROM $(\mathrm{p}=0.28)$. unpaired t-test revealed no statistically significant difference when comparing both groups for pain $(p=0.08)$, SPADI $(p=0.1)$, shoulder flexion $\operatorname{ROM}(\mathrm{p}=0.16)$. shoulder abduction ROM $(\mathrm{p}=0.54)$ and shoulder external rotation ROM $(p=0.35)$.

\section{Discussion}

This study intended to assess the efficacy of ESWT for frozen shoulder in perimenopausal women, many studies investigated the efficacy of ESWT on several musculoskeletal disorders $[17,19,22,28]$. however, studies investigating efficacy of ESWT on frozen shoulder remain rare and require further studies on specific set of populations as well. This paper addressed this issue by focusing on a specific population represented by diabetic perimenopausal women which so far lacking in the scientific literature.

Adhesive capsulitis disrupts patient's quality of life; usually patients suffer from shoulder pain, limited ROM, and sleep disturbances in severe cases. Different techniques are used in physical therapy for treating diabetic frozen shoulder which include mobilization techniques, psychotherapy, manual therapy, thermotherapy, ultrasound therapy, etc. [35]. ESWT therapy is a new treating method, which is a non-surgical treatment method. Studies reported that combination of ESWT with physical exercise had beneficial impacts on FS $[30,35]$. In this study, we found that pain intensity was decreased, and function and shoulder ROM were significantly increased in both groups in case of intragroup comparison. However, no significant differences could be obtained when comparing both groups in intergroup level. Chen et al., [9] reported statistically significant differences in shoulder ROM measurements on 40 patients with adhesive capsulitis after the application of ESWT. Consistent results were reported by Muthukrishnan et al., [30] who evaluated the effectiveness of ESWT versus ultrasound therapy in patients with diabetic frozen shoulder, they combined both treatment modalities with joint mobilization and therapeutic exercises. They obtained positive impact of ESWT combined with exercises and joint mobilization on pain scores in patients with diabetic frozen shoulder. Their results highlight the significance of adding exercises and manual therapy techniques to ESWT in treatment of frozen shoulder. However, Lee et al. [21] used only ESWT on 30 patients suffering from adhesive capsulitis regarding pain level and shoulder ROM and reported that the pain decreased, and the ROM increased in the intragroup level, but the ESWT group had lower level of pain and higher ROM in intergroup level, suggesting that ESWT, as an effective intervention to reduce pain and improve the ROM in adhesive capsulitis patients.

These results reflected the beneficial effect of adding exercise therapy plus ESWT on diabetic women suffering from shoulder adhesive capsulitis. Application of stretching exercise can reduce the additional tensile loads developed around the non-contractile connective tissue of the shoulder joint and produce inhibition of the muscular contractile elements by the Golgi tendon organ that causes reflex relaxation of the muscle. This can enhance muscle lengthening with less tension [6].

Meanwhile, joint mobilization also contributes to elicited golgi tendon organ activity which helps to inhibit tight muscles around the shoulder joint [23]. Mangus et al. highlighted the analgesic assets of joint mobilization based on its neurophysiological effects through stimulation of mechanoreceptors and inhibition of nociceptors [24]. Another positive effect of joint mobilization is its ability to breakdown adhesions, realign collagen, increase fiber gliding through particular movements stressing the capsular tissues and increase fluid exchange within the joint. [2]

Vahdatpour et al., [39] found a significant reduction in pain and disability scores of SPADI, as well as a significant improvement in shoulder ROM in patients with frozen shoulder after 4 weeks of shock wave therapy. The results of the study of Alarab et al. confirmed that the combination of ESWT plus exercising help to reduce pain and improve shoulder ROM in patients with frozen shoulder [3].

One reason why VAS scores declined is the metabolic effects of ESWT that alter the cell metabolic rate [34]. In addition, the ESWT has a significant contributory factor to healing of the tissues through secretion of angiogenic substances that enhance blood flow to the region [16]. Also, application of ESWT with its fine repetitive stimulation can suppress the activity of nociceptors [22].

According to the results of the current study, the study group showed a significant increase in the in all directions (flexion, abduction and external rotation) and significant reduction in VAS and SPADI which are consistent with the results of Do Moon et al. who recommended oscillatory and sustained mobilization techniques for treating patients with frozen shoulder due to their positive effects on the patient's pain and shoulder ROM [29].

\section{Limitations}

The generalizability of these results is subject to certain limitations. For instance, uncontrolled diabetes mellitus for long time would influence the extent of capsular fibrosis. This should be considered in future research. In addition, the lack of long term follows up failed to obtain additional results. Although the study used valid established measurements, adding an objective measurement 
such as magnetic resonance imaging would have empowered the results. The strength of this paper is that it was the first work studying the effect of shock wave on frozen shoulder in perimenopausal women.

\section{Conclusion}

The combination of shockwave therapy and standard physical therapy program for 4 weeks has a positive effect on reducing pain, functional disability and increasing shoulder ROM in diabetic perimenopausal women suffering from $\mathrm{AC}$.

Conflict of interest: Authors state no conflict of interest.

\section{References}

1. Adel S.M., ElAzm S.A.N., Abdel-Raof N.A., Ahmed K.T. (2013) Shock Wave versus Phonophoresis in Treatment of Diabetic Frozen Shoulder. J. Med. Res. Pract., 2: 148-156.

2. Agarwal S., Raza S., Moiz J.A., Anwer S., Alghadir A.H. (2016) Effects of two different mobilization techniques on pain, range of motion and functional disability in patients with adhesive capsulitis: a comparative study. J. Phys. Ther. Sci., 28: 3342-3349. DOI: 10.1589/jpts.28.3342.

3. Alarab A. (2018) Shock Wave Therapy and Ultrasound Therapy plus Exercises for Frozen Shoulder Joint Clients. J. Public Health Policy Plann., 1: 180010.

4. Bang M.D., Deyle G.D. (2000) Comparison of supervised exercise with and without manual physical therapy for patients with shoulder impingement syndrome. $J$. Orthop. Sports Phys. Ther, 30: 126-137. DOI: 10.2519/ jospt.2000.30.3.126.

5. Blake J.M., Contestabile E., Fortier M. (2001) Evaluation, Decision-Making and Follow-up. Journal SOGC, 23: 1214-1220. DOI: 10.1016/s0849-5831(16)30969-7.

6. Brotzman, S.B., Manske R.C. (2012) Clinical orthopaedic rehabilitation: An evidence-based approach-Third edition. J. Can. Chiropr. Assoc., 56: 234.

7. Cao D.-Z., Wang C.-L., Qing Z., Liu L.-D. (2019) Effectiveness of extracorporeal shock-wave therapy for frozen shoulder: A protocol for a systematic review of randomized controlled trial. Medicine, 98: e14506. DOI: 10.1097/MD.0000000000014506.

8. Chan H.B.Y., Pua P.Y., How C.H. (2017) Physical therapy in the management of frozen shoulder. Singapore Med. J., 58: 685-689. DOI: 10.11622/smedj.2017107.

9. Chen C.-Y., Hu C.-C., Weng P.-W., Huang Y.-M., Chiang C.-J., Chen C.-H., Tsuang Y.-H., Yang R.-S., Sun J.-S., Cheng C.-K. (2014) Extracorporeal shockwave therapy improves short-term functional outcomes of shoulder adhesive capsulitis. J. Shoulder Elbow Surg., 23: 1843-1851. DOI: 10.1016/j.jse.2014.08.010.

10. Cheng X., Zhang Z., Xuanyan G., Li T., Li J., Yin L., Lu M. (2017) Adhesive capsulitis of the shoulder: evaluation with US-arthrography using a sonographic contrast Agent. Sci. Rep., 7: 5551. DOI: 10.1038/s41598-01705491-x.

11. Contestabile E., Derzko C. (2001) Canadian consensus on menopause and perimenopause. J. Obstet. Gynaecol. Can., 23: 836-841.

12. Dashottar A., Costantini O., Borstad J. (2014) A comparison of range of motion change across four posterior shoulder tightness measurements after external rotator fatigue. Int. J. Sports Phys. Ther, 9: 498-508.

13. Dedicação A.C., Sato T. de O., Avila M.A., Moccellin A.S., Saldanha M.E.S., Driusso P. (2017) Prevalence of musculoskeletal pain in climacteric women of a Basic Health Unit in São Paulo/SP. Rev. Dor., 18: 212-216. DOI: 10.5935/1806-0013.20170104.

14. Ewald A. (2011) Adhesive capsulitis: a review. Am. Fam. Physician, 83: 417-422.

15. Grant S., Aitchison T., Henderson E., Christie J., Zare S., McMurray J., Dargie H. (1999) A comparison of the reproducibility and the sensitivity to change of visual analogue scales, Borg scales, and Likert scales in normal subjects during submaximal exercise. Chest, 116: 1208-1217. DOI: 10.1378/chest.116.5.1208.

16. Hammer D.S., Rupp S., Ensslin S., Kohn D., Seil R. (2000) Extracorporal shock wave therapy in patients with tennis elbow and painful heel. Arch. Orthop. Trauma Surg., 120: 304-307. DOI: 10.1007/s004020050470.

17. Huisstede B.M.A., Gebremariam L., van der Sande R., Hay E.M., Koes B.W. (2011) Evidence for effectiveness of Extracorporal Shock-Wave Therapy (ESWT) to treat calcific and non-calcific rotator cuff tendinosis-a systematic review. Man. Ther, 16: 419-433. DOI: 10.1016/j. math.2011.02.005.

18. Jankovic D., van Zundert A. (2006) The frozen shoulder syndrome. Description of a new technique and five case reports using the subscapular nerve block and subscapularis trigger point infiltration. Acta Anaesthesiol. Belg., 57: 137-143.

19. Jeon, J.H., Jung Y.J., Lee J.Y., Choi J.S., Mun J.H., Park W.Y., Seo C.H., Jang K.U. (2012) The effect of extracorporeal shock wave therapy on myofascial pain syndrome. Ann. Rehabil. Med., 36: 665-674. DOI: 10.5535/ arm.2012.36.5.665.

20. Khadilkar S.S. (2019) Musculoskeletal disorders and menopause. J. Obstet. Gynaecol. India, 69: 99-103. DOI: 10.1007/s13224-019-01213-7.

21. Lee S., Lee S., Jeong M., Oh H., Lee K. (2017) The effects of extracorporeal shock wave therapy on pain and range of motion in patients with adhesive capsulitis. J. Phys. Ther. Sci., 29: 1907-1909. DOI: 10.1589/jpts.29.1907. 
22. Loew M., Daecke W., Kusnierczak D., Rahmanzadeh M., Ewerbeck V. (1999) Shock-wave therapy is effective for chronic calcifying tendinitis of the shoulder. J. Bone Joint Surg. Br., 81: 863-867. DOI: 10.1302/0301-620x.81b5.9374.

23. Lundberg A., Malmgren K., Schomburg E.D. (1978) Role of joint afferents in motor control exemplified by effects on reflex pathways from Ib afferents. J. Physiol., 284: 327-343. DOI: 10.1113/jphysiol.1978.sp012543.

24. Mangus B.C., Hoffman L.A., Hoffman M.A., Altenburger P. (2002) Basic principles of extremity joint mobilization using a Kaltenborn approach. J. Sport Rehabil., 11: 235-250. DOI: 10.1123/jsr.11.4.235.

25. Maricar N., Shacklady C., McLoughlin L. (2009) Effect of Maitland mobilization and exercises for the treatment of shoulder adhesive capsulitis: a single-case design. Physiother. Theory Pract., 25: 203-217. DOI: 10.1080/09593980902776654.

26. McClure S.K., Adams J.E., Dahm D.L. (2005) Common Musculoskeletal Disorders in Women. Mayo Clin. Proc., 80: 796-802. DOI: 10.1016/S0025-6196(11)61534-6.

27. Mehta A., Nilima B. (2018) Passive Stretching Exercises Versus Mulligan Mobilization with Movement for Pain, Range of Motion \& Function in Patients of Adhesive Capsulitis : A Comparative Study. Int. J. Physiother. Res., 6: 2784-2790. DOI:10.16965/ijpr.2018.145.

28. Moayednia A., Haghdani S., Khosrawi S., Yousefi E., Vahdatpour B. (2014) Long-term effect of extracorporeal shock wave therapy on the treatment of chronic pelvic pain syndrome due to non bacterial prostatitis. J. Res. Med. Sci., 19: 293-296.

29. Moon G.D., Lim J.Y., Kim D.Y., Kim T.H. (2015) Comparison of Maitland and Kaltenborn mobilization techniques for improving shoulder pain and range of motion in frozen shoulders. J. Phys. Ther. Sci., 27: 1391-1395. DOI: $10.1589 /$ jpts.27.1391.

30. Muthukrishnan R., Rashid A.A., Al-Alkharji F. (2019) The effectiveness of extracorporeal shockwave therapy for frozen shoulder in patients with diabetes: randomized control trial. J. Phys. Ther. Sci., 31: 493-497. DOI: 10.1589/jpts.31.493.

31. Noten S., Meeus M., Stassijns G., Van Glabbeek F., Verborgt O., Struyf F. (2016) Efficacy of Different Types of Mobilization Techniques in Patients With Primary Adhesive Capsulitis of the Shoulder: A Systematic Review. Arch. Phys. Med. Rehabil., 97: 815-825. DOI: 10.1016/j. apmr.2015.07.025.
32. Park G.-Y. (2019) Role of ultrasonography in diagnosis and treatment of frozen shoulder. J. Rheum. Dis., 26: 149-155. DOI: 10.4078/jrd.2019.26.3.149.

33. Robinson C.M., Seah K.T.M., Chee Y.H., Hindle P., Murray I.R. (2012) Frozen shoulder. J. Bone Joint Surg. Br., 94: 1-9. DOI: 10.1302/0301-620X.94B1.27093.

34. Seidl M., Steinbach P., Wörle K., Hofstädter F. (1994) Induction of stress fibres and intercellular gaps in human vascular endothelium by shock-waves. Ultrasonics, 32: 397-400. DOI: 10.1016/0041-624x(94)90111-2.

35. Seyam M.K., Moubarak E.E., Shaik A.R. (2018) The Effect of Extracorporeal Shock Wave Therapy for Patients with Diabetic Frozen Shoulder. Majmaah J. Heal. Sci., 6: 40-49. DOI: 10.5455/mjhs.2018.02.006.

36. Shehri A.A., Almureef S.S., Khan S., Shamsi S. (2018) Efficacy of Maitland Mobilization in Frozen Shoulder. Eur. J. Biomed. Pharm., 5: 22-27.

37. Sözen T., Başaran N.C., Tinazli M., Özişik L. (2018) Musculoskeletal problems in diabetes mellitus. Eur. J. Rheumatol., 5: 258-265. DOI: 10.5152/eurjrheum.2018.18044.

38. Tveitå E.K., Sandvik L., Ekeberg O.M., Juel N.G., BautzHolter E. (2008) Factor structure of the Shoulder Pain and Disability Index in patients with adhesive capsulitis. BMC Musculoskelet Disord., 9: 103. DOI: 10.1186/14712474-9-103.

39. Vahdatpour B., Taheri P., Zade A.Z., Moradian S. (2014) Efficacy of extracorporeal shockwave therapy in frozen shoulder. Int. J. Prev. Med., 5: 875-881.

40. World Health Organization (2021) COVID-19: Occupational health and safety for health workers. Accessed on July 26, 2021. https:/www.who.int/publications/i/item/ WHO-2019-nCoV-HCW_advice-2021.1

41. Yokota M., Makita K., Hirasawa A., Iwata T., Aoki D. (2016) Symptoms and effects of physical factors in Japanese middle-aged women. Menopause, 23: 974-983. DOI: $10.1097 / \mathrm{GME} .0000000000000660$.

\section{Received 16.02.2021 \\ Accepted 12.10.2021}

\section{(C) University of Physical Education, Warsaw, Poland}

\section{Acknowledgments}

The authors are grateful for each individual who contributed and shared their time to this study. 